\title{
UAV Formation Occupying Technology Research Collaborative
}

\author{
Yibo $\mathrm{Li}^{1}$, Xiaonan Guo ${ }^{1}$, Wei Chen ${ }^{2}$ and Yi Wang ${ }^{2}$ \\ School of Automation, Shenyang Aerospace University, Liao Ning, China. \\ AVIC Chengdu aircraft industrial (group) CO,LTD, Chengdu, China \\ liyibo_sau@163.com,gxn_03_30@163.com,scjjwy@21cn.com
}

\begin{abstract}
Combined with our aircraft combat capability, this paper aims at the unmanned aerial vehicles automatic attack process of single target, in accordance with the principle of maximum killing the enemy and establish the model of target threat based on the situation of two sides. According to the importance of our members, defense model, and the enemy motion trajectory with the threat model and the cooperative attack and defense model of pseudo tracking site, our cooperative tracking of pseudo trace point to obtain the best accounts for the site and to fight against the target. The simulation results show that, the occupied position is a rapid and effective method which could lead to the greatest degree of destroy to enemy aircraft.
\end{abstract}

Keywords: threat evaluation; collaborative defense; collaborative formations; occupy position

\section{Introduction}

Air war will shift from the human combat to UAV combat gradually in the 21st century. In order to guarantee the success rate of combat, the U.S. military field has been studying the "air occupying technology" which will also be the mainstream trends of UAV combat among various countries in the future.

At present, domestic and overseas have done some valuable research in UAV attacking occupying Technology, literature[1] applied minimum principle to solve the UAV attack placeholder paragraph should automatically fly trajectory ,designed UAV automatic attack placeholder track technology. literature [2] proposed UCAV close combat placeholder strategy and methods according to the existence of harsh conditions and the target missile launch success rate dropped when maneuvering and application limitations countermeasures occupying discrete method based on the larger issues in the pre- war occupancy tracking method.[3]Using fuzzy neural network method realized the forecast to the target maneuvering intention, put forward fuzzy neural network method of the fighter air combat occupying. But they are one -on-one study or many- UAV technology footprint, there is no consideration on the basis of threat assessments.

In this paper, we firstly create a pseudo- point motion tracking model in allusion to UAV coordinated attacks single target attack process, then ,according to the pseudo- point motion tracking model combines threat model, cooperative attack and defense model and validity of the pseudo- tracking placeholder point. Thus, our formations collaborative tracking pseudo- tracking points to the best placeholder points and hit on the target.

\section{Pseudo- Point Motion Tracking Model}

\subsection{Radar Instantaneous Field of view Relative to the Model}

If $\mathrm{UAV}$ is under the radar detection range 
$m=\left\{\begin{array}{rr}1 & M C\left(\theta_{t}+\alpha / 2\right)>M C(\xi+\pi) \cap M C\left(\theta_{t}-\alpha / 2\right)<M C(\xi-\pi) \cap M C(\xi+\pi)>\theta_{T} \\ -1 & M C\left(\theta_{t}+\alpha / 2\right)>M C(\xi+\pi) \cap M C\left(\theta_{t}-\alpha / 2\right)<M C(\xi-\pi) \cap M C(\xi+\pi) \leq \theta_{T} \\ 0 & M C\left(\theta_{t}+\alpha / 2\right) \leq M C(\xi+\pi) \cap M C\left(\theta_{t}-\alpha / 2\right) \geq M C(\xi-\pi)\end{array}\right.$

Among them, $\theta_{t}$ is the azimuth of enemy, $\xi$ is the sight inclination, $M C(x)$ is the lord periodic function; $m=1 /-1 / 0$ denotes the UAV located in the aircraft radar left no right detection area.

\subsection{Pseudo- tracking Point Coordinate Calculation}

UAV operational relative motion model:

$$
\begin{aligned}
& D=\sqrt{\left(X_{T}-X\right)^{2}-\left(Y_{T}-Y\right)^{2}} \\
& \xi=A t\left[\left(Y_{T}-Y\right) /\left(X_{T}-X\right)\right] \\
& \dot{D}=V_{T} \cos \left(\xi-\theta_{T}\right)-V \cos (\xi-\theta) \\
& \omega=\left[V \sin (\xi-\theta)-V_{T} \sin \left(\xi-\theta_{T}\right)\right] / D
\end{aligned}
$$

Among them, $\omega$ is the sight inclination of the angular velocity. $X, Y, \theta, V$ is the UAV information; $X, Y, \theta_{T}, V_{T}$ is the enemy information.

2.2.1. When the UAV is Not in the Enemy Plane's Range of Detection Angle, the Calculation of Pseudo- tracking: Suppose the pseudo tracking point is outside the boundaries of the enemy radar detection, the calculation of the direction angle is

$$
\theta^{\prime}= \begin{cases}M C\left(\theta_{r}+\beta \frac{\alpha}{2}\right) & m=1 \\ M C\left(\theta_{r}-\beta \frac{\alpha}{2}\right) & m=-1\end{cases}
$$

Among them, $\beta \geq 1$.

Calculation of the pseudo- tracking coordinates

$$
\begin{aligned}
& X^{\prime}=X_{r}+D^{\prime} \cos \theta \\
& Y^{\prime}=Y_{r}+D^{\prime} \cos \theta
\end{aligned}
$$

When the UAV is in the enemy plane's range of detection angle and not in the scope range, $D$ is the most radar detection range of enemy plane; when the UAV is in the enemy plane's range of detection angle and also in the scope range, $D$ is the $K, D$, that

$$
D^{\prime}= \begin{cases}D_{R \max } & |m|=1 \cap D>D_{R \max } \\ K, D & |m|=1 \cap D<D_{R \max }\end{cases}
$$

2.2.2. When the UAV is Not in the Enemy Plane's Range of Detection Angle, the Calculation of the Pseudo- tracking:

$$
X^{\prime}=\left\{\begin{array}{cc}
X_{r} \quad & m=0 \cap|M c(\theta)-M c(\theta)| \geq \frac{\pi}{2} \\
X+\left(D-D_{M}\right) \cos \xi & m=0 \cap|M c(\theta)-M c(\theta)|<\frac{\pi}{2}
\end{array}\right\}
$$

$D_{M}$ is the UAV missile range. 
Through the analysis above, we found that we need a law to guide the UAV to a pseudo- tracking point quickly and accurately. In this paper, we applied the variable structure guidance law proposed in literature[4] to lead the UAV to pseudo- tracking points.

\section{Threat Assessment Model}

Air combat situation assessment method is divided into two categories, parameter method and non-combat situation assessment parameter method.

In this paper, a non- parametric method, as is shown in Figure 1:

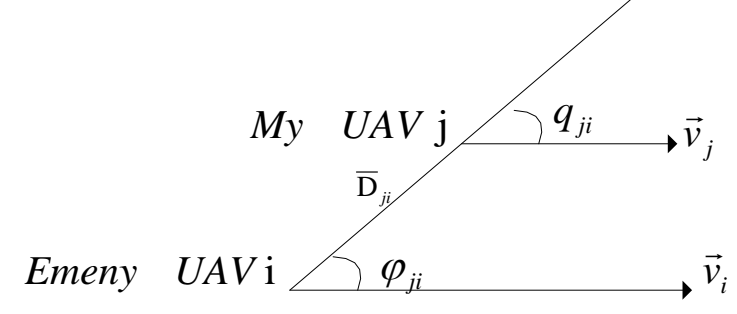

\section{Figure 1. Schematic Combat Situation}

Among them, $\left|\bar{V}_{i}\right|$ is my UAV's velocity vector; $\left|\bar{D}_{j i}\right|$ is the target distance; $\left|V_{j_{j}}\right|$ is the velocity vector of enemy UAV; $\varphi_{j i}$ is the angle for the enemy, that is to say the line angle between UAV and the target heading machine.

\subsection{Angle Advantage}

Position of the aircraft in the whole UAV coordinated attacks occupying plays a vital role, a suitable location can be a good potential to overcome the lack of performance on their own .So that the enemy aircraft could not play its due role and the role of the enemy aircraft was restrained. Among these, the angular relationship between the UAV of both sides will directly affect the use of motor strategy and missile weapons.

The angular advantage of our aircraft relative to enemy aircraft is defined as:

Taji $=\left(\left|q_{j i}\right|+\left|\varphi_{j i}\right|\right) / 2 \pi$

\subsection{Speed Advantage}

Speed is also one of the factors that have a direct impact on the situation advantage. In the attack placeholder, the UAV who first reaches the desired speed will have more advantages than others, would have greater advantages in the attack placeholder.

$T v_{i}=\left\{\begin{array}{c}-0.1 v_{i}+1, v_{i} \leq 0.6 \\ 0.94 e^{\left(\left(0.6-v_{i}\right) / 0.4\right)}, 0.6<v_{i}<1.5 \\ 0.1 e^{\left(1.5-v_{i}\right)}, v_{i}>1.5\end{array}\right.$

\subsection{Performance Advantages}

$\operatorname{Tr}_{i}=\left\{\begin{array}{c}-0.05 r_{i} / r m t+1, r_{i}<r m t \\ 0.45\left(r m t-r_{i}\right) /(r r t-r m t)+0.95, r m t \leq r_{i} \leq r r t \\ 0.5 e^{\left(r t-r_{i}\right)}, r_{i}>r r t\end{array}\right.$

In the state, $r m t$ is the $j$ flight enemy missiles, $r r$ is the enemy maximum detection distance. 


\subsection{Distance Advantage}

Taking into account the influence of carrier aircraft's height on the air-to-air missile, we introduce an influence coefficient, where $m=(H+6500) / 6500$. Because inside the radar area, radar target detection probability is $P=e^{\left(-0.1625 D / D_{R_{\max }}\right)}$, so the distance advantage increases with the distance function decreases exponentially.

$$
T_{S D}=\left\{\begin{array}{c}
\left(1-m^{2}\right) 0.1839 e^{\frac{D-r r t}{r t}}, D \geq r r t \\
\left(1-m^{2}\right) 0.5 e^{-\frac{D-r m t}{r t-m t}}, m r m t \leq D<r r t \\
\left(1-m^{2}\right), D<m r m t
\end{array}\right.
$$

\subsection{UAV Overall Threat Assessment Decision Model}

According to expert opinion, the establishment of UAV twenty-two compared with each other pairwise comparison matrix. Each time, we take two UAVs $U_{i}$ and $U_{j}$, use $a_{i j}$ show the effect of the $U_{i}$ and $U_{j}$ of $p$, In the comparison process, TL Satie, who proposed using the 1-9 scale, which is defined as follows in Table 1:

Table 1. 1-9 Scale Definition Table

\begin{tabular}{c|c}
\hline scale $a_{i j}$ & meaning \\
\hline $\begin{array}{c}\text { scale } a_{i j} \\
\text { scale } a_{i j}\end{array}$ & $C_{i}$ and $C_{j}$ are the same effects \\
scale $a_{i j}$ & $C_{j}$ with $C_{i}$ a slightly stronger impact \\
scale $a_{i j}$ & $C_{j}$ with $C_{i}$ a strong impact \\
scale $a_{i j}$ & $C_{i}$ and $C_{j}$ are obviously strong impact \\
$2,4,6,8$ & $\begin{array}{c}C_{i} \text { and } C_{j} \text { effect the absolute intensity } \\
\text { effect of the ratio between } C_{i} \text { and } C_{j} \\
\text { appeal adjacent two levels } \\
1,1 / 2, \ldots, 1 / 9\end{array}$ \\
$C_{i}$ and $C_{j}$ the ratio of the effect of the \\
opposite ( "strong to weak" )
\end{tabular}

Comparison of the results of all of the layers constituting the PC judgment matrix as shown in Table 2:

Table 2 P-C Layer Judgment Matrix

\begin{tabular}{|c|c|c|c|c|}
\hline$a_{i j}$ & $C_{1}$ & $C_{2}$ & $C_{3}$ & $C_{4}$ \\
\hline$C_{1}$ & 1 & $a_{12}$ & $\cdots$ & $\cdots$ \\
\hline$C_{2}$ & $\frac{1}{a_{12}}$ & 1 & & \\
\hline$C_{3}$ & $\cdots$ & & 1 & \\
\hline$C_{4}$ & $\cdots$ & & & 1 \\
\hline
\end{tabular}


Calculate the approximate feature layer $P-C$ judgment matrix vector summation method with $H=\left(\lambda_{1}, \lambda_{2}, \lambda_{3}, \lambda_{4}\right)$

Policy makers choose to maneuver during combat is based on UAV threat index, different combat forms, factors affecting operations will be different:

Thread ${ }_{i}=\lambda_{1} T a_{i}+\lambda_{2} \operatorname{Tr}_{i}+\lambda_{3} T v_{i}+\lambda_{4} T_{S D}$

Thread ${ }_{i}$ is the UAV threat index, $\lambda_{i}$ is the weight of each threat index. In front of each factor on the type of threat index multiplied by the weight of each threat index $\lambda_{1}, \lambda_{2}$, $\lambda_{3}$ and $\lambda_{4}$, weight depends on the preference of decision makers, Finally, the situation can be constructed UAV aerial threat index .

So Thread $=\sum$ Thread $i$ is all of enemy drones and threatened.

\subsection{Attack Effectiveness Index}

One factor which also need to be considered is the combat capability of enemy aircraft drones. In this paper, we choose the logarithmic law which is often used in combat [6] and select seven main parameters to assess the fighter-air combat capability. Including fighters themselves flexibility, weaponry, target detection capability, steering performance, survivability, and practical maximum range of electronic warfare capabilities. Integrated combat capability of determining the number of law to obtain air combat capability factor:

$$
C=\left[\operatorname{InB}+\operatorname{In}\left(\sum A_{1}+1\right)+\operatorname{In}\left(\sum A_{2}\right)\right] \varepsilon_{1} \varepsilon_{2} \varepsilon_{3} \varepsilon_{4}
$$

Among them, $B$-mobility parameters, $A_{1}$ - Fire parameters, $A_{2}$ - The ability to detect the target parameters, $\varepsilon_{1}$ - Manipulate the performance factor , $\varepsilon_{2}$ - Viability factor, $\varepsilon_{3}$ Range factor, $\varepsilon_{4}$ - Electronic warfare capability factor.

3.6.1. Single Occupancy Performance Index: Assuming combat aircraft performance index is composed of two parts, air-to-ground performance index and air-to-air performance index. Both share in the total index performance index based on the proportion of courses required for aircraft task given [7].Let $C$ is the air performance index, $D$ is the ground performance for the index, $E$ is the total performance index

$E=\alpha_{1} C+\alpha_{2} D$

Among them, $\alpha_{1}, \alpha_{2}$ is the air-to-air combat and air-to-ground combat missions partition coefficient, both equals is the 1 , we only consider the so -air combat effectiveness is 0.

3.6.2. Cooperative Air Combat Effectiveness Index: Combat fighting index can be calculated:

$\left\{\begin{array}{c}F_{R_{i}}=E 1 / \bar{n}\left(n_{r}\right)^{x} \\ F_{B_{j}}=E_{B_{j}} 1 / \bar{n}\left(n_{b}\right)^{x}\end{array}\right.$

Among them, $F_{R_{i}}$ is our UAV $i$ 's air combat capability index. $F_{B_{j}}$ is the enemy airplane $j$ 's air combat capability index, $\bar{n}$ is the average number of combat aircraft; $n_{r}$ is the number of our combat UAV; $n_{b}$ is the number of enemy combat aircraft ; $x$ is the power of number associated with the number of combat aircraft, it can be decided by the number of war planes of both sides. 


\subsection{The Overall Threat Index Model}

Due to differences in air combat situation index and air combat capability index is relatively large, data processing is needed at first. Here we take the relative value of air $\operatorname{index} T_{i j}$ :

$T_{i j}=\left(F_{R_{i}}-F_{B_{j}}\right) / F$

$F$ is the maximum value of all war combat aircraft performance index.

Synthesize the effects of Air combat capability index and air combat situation index, the total threat index is:

$W_{i}=b_{1} * T_{i j}+b_{2} *$ Thread

Among them, $W_{i}$ is the enemy aircraft threat index, $b_{1}, b_{2}$ is the weights $\left(b_{1} \geq 0\right.$, $b_{1} \geq 0$ ) ,generally make $b_{1}=0.5 ; b_{2}=0.5$.

\section{Validity of the Model Collaborative Defense}

The distance between UAVs is an important part of the UAV defense system. Distance is too large or too small will affect directly the UAV collaborative defense capability.

The distance between the two planes is:

$D_{i j}=\sqrt{\left(x_{i}-x_{t}\right)^{2}-\left(y_{i}-y_{t}\right)^{2}}$

For the UAV, its defensive capabilities index is:

Defense $_{i}=\sum_{i, j=1}^{3} K\left(D_{i, j}-D_{p}\right)$

Among them, $k$ is a appropriate parameter, $D_{p}$ is the optimum distance between the

UAVs collaborative defense.

So the collaborative defense of all UAVs is:

Defense $=\sum_{i=1}^{3} r_{i}$ Defense $\quad$ i

Paper [7] proposed threat assessment and combat effectiveness model, but it did not put it in defense validity model. In order to unify these three models, this paper takes the following normalization process.

Max $F_{i}=\eta_{1} w_{i}+\eta_{2}$ Defense
$r \geq 0$
$s t .\left\{\begin{array}{c}\theta \in[0,2 \pi] ; \varphi \in[0, \pi] \\ v_{1,2,3} \in[0,0.6] ; v_{4} \in[0,1.2]\end{array}\right.$

Among them, $\eta_{1}, \eta_{2}$ is attack weight and defense weight, it can be obtained by structured with $P-C$ judgment matrix. Adjust the weights to be focused on the tactical defensive or attack based.

In order to analyze the probability of destroying enemy planes clearly, according the combat situation, set $P \geq 0.550$ is the maximum probability which enemy aircraft can not escape, application of the linear function method for data normalization, for sample data $W_{i}, i=1,2, \ldots, N$,

$P(x)=\frac{W(x)-\min \left(W_{i}\right)}{\max \left(W_{i}\right)-\min \left(W_{i}\right)}, x=1,2, \ldots, N$

The point obtained when $\mathrm{P}(\mathrm{x})$ is greater than 0.5 is the best attack placeholder point. 


\section{Multi- UAV Formation Attack Footprint Model}

In order to obtain the most favorable placeholder point, we need to get enemy aircrafts' real-time status information. And our three UAVs should avoid a collision in the process of flying to the best placeholder point. So we must ensure consistency in time and space. This section establishes UAV collaborative motion model firstly, and then builds track occupying algorithm model.

\subsection{UAV Motion Model}

According to the UAV particle motion model which is proposed in literature [7], the UAVs are defined as conducting operations at the same height. It is to considerate be within the two-dimensional plane UAV particle motion, the motion equation is as follows:

$\left\{\begin{array}{c}x_{i}(k+1)=x_{i}(k)+t v_{i} \cos \phi_{i}(k) \\ y_{i}(k+1)=y_{i}(k)+t v_{i} \sin \phi_{i}(k) \\ \phi_{i}(k+1)=\phi_{i}(k)+t u_{i}(k)\end{array}\right.$

In the above formulas, $v_{\max }, v_{\min }$ denote the UAV maximum and minimum flight speed respectively, $a_{\text {max }}, a_{\text {min }}$ is the maximum and minimum UAV flight acceleration accordingly, $\phi_{\text {max }}$ is heading angle constraint UAVs.

\subsection{Establishment of Multi- UAV Formation Structure}

In this paper, we established commonly used Leader-Follower model in Figure 3.2, UAV2 is the UAV formation collar machine, two others form a triangle formation as wingman.

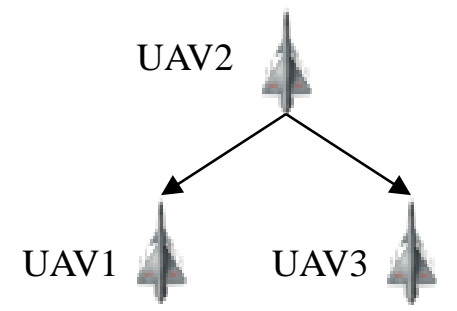

Figure 2. Leader-Follower Formation Model

According to the design method of formation in literature [8], UAV formation algorithm formula to achieve the above formation is as follows:

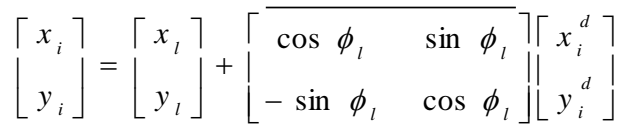

Among, $x_{i}, y_{i}$ indicate the position coordinates of $i$ th UAV, $x_{l}, y_{l}$ indicate the position coordinates of the leader, $\phi_{l}$ represents the heading angle of a leader in UAVs, $x_{i}^{d}, y_{i}^{d}$ are location coordinates to achieve the desired formation.

\subsection{Establish Fleet Tracking Trajectory Model}

We can get the pseudo tracking occupying track of enemy planes by above method. Furthermore, combined with our multi- UAV fleet tracking and sports consistency algorithm, we quickly formed a multi- UAV fleet tracking enemy occupying pseudopoint tracking. Then attack enemy in the most powerful placeholder point found by above threat assessment, causing the maximum extent of damage to enemy aircraft. 
Motion equations of the collar machine in our UAV formation:

$$
\left\{\begin{array}{c}
x_{j 1}=x_{i 1}+t v_{i 1} \cos \phi_{i 1}+x_{j 1}^{d} \cos \phi_{i 1}+y_{j 1}^{d} \sin \phi_{i 1}+\exp (-a t) \\
y_{j 1}=y_{i 1}+t v_{i 1} \cos \phi_{i 1}-y_{j 1}^{d} \cos \phi_{i 1}+y_{j 1}^{d} \cos \phi_{i 1}+\exp (-a t) \\
\phi_{j 1}=\phi_{i 1}+t u_{i 1}
\end{array}\right.
$$

The above equations consists of two parts , the first part is the particle motion model of UAV, subscript il represents enemy aircraft, subscript $j l$ represents the collar machine of our UAV formation, The latter part is a model to calculate the formation of our enemy UAV formation collar machine and maintain a certain position, Wherein $a, b$ are the coordinates of a desired position of the machine of our collar UAV formation ( optimal placeholder point),the final error vector is a regression factor based on the consistency algorithm in [8], $a$ is a regression constant, the larger a is , it disappears more quickly.

\subsection{Establishment of Attack Occupying}

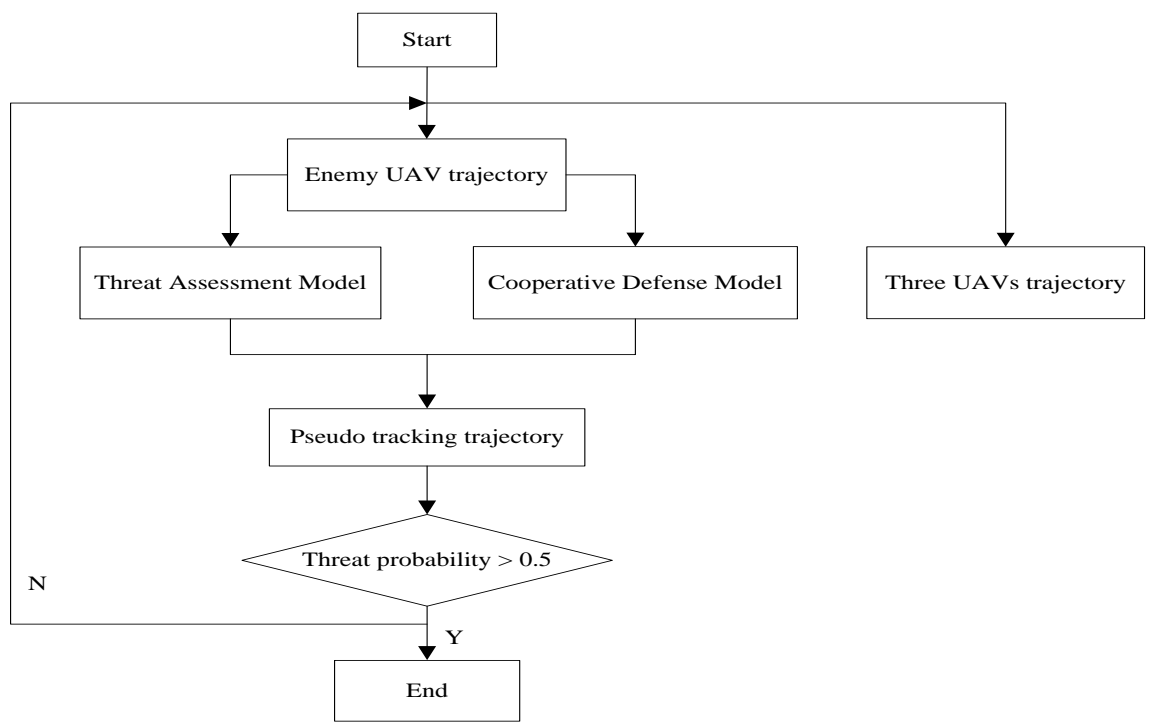

Figure 3. Flowchart Placeholder Attack

\section{A Numerical Example}

\subsection{Pseudo- point Trajectory Tracking}

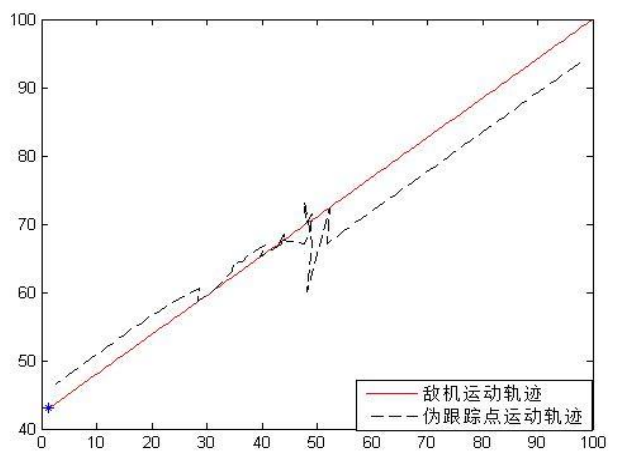

Figure 4. Pseudo-point Trajectory Tracking 


\subsection{Formation Flying Model Implementation}

According to the UAV formation flying algorithm in formula 4, we achieved the formation structure shown in Figure 5 and 6.

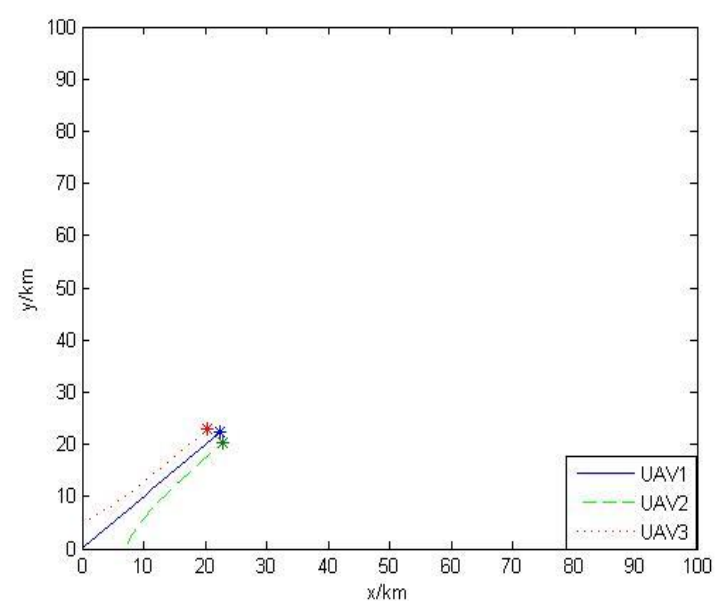

Figure 5. Leader-Follower Formation Flying

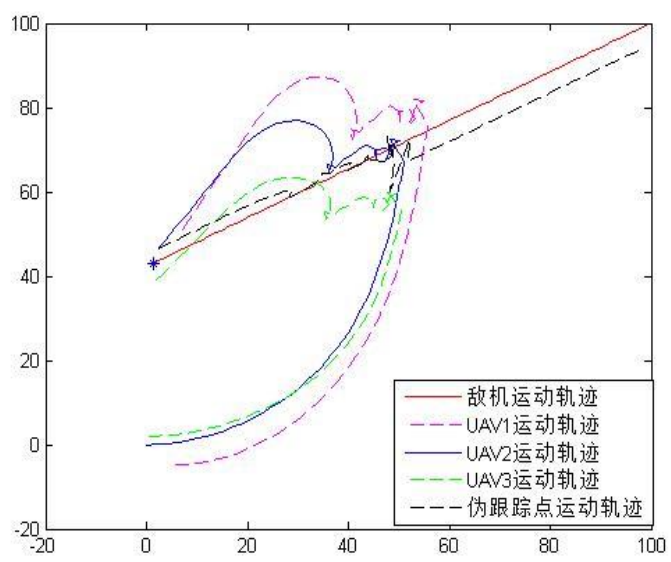

Figure 6. Formation Placeholder Model

\subsection{Formation Placeholder Implementations}

We have three UAVs, the initial position is $\operatorname{UAV} 1(0,5) \mathrm{km}, \operatorname{UAV} 2(0,0) \mathrm{km}$, UAV3 $(7,0) \mathrm{km}$, the initial flight speed is $4500 \mathrm{~m} / \mathrm{s}$, move with a constant velocity; There is only one enemy aircraft, the initial position is $(50,50) \mathrm{km}$, its initial flight speed is $5300 \mathrm{~m} / \mathrm{s}$, also moves with a constant velocity . Our UAV aerial index is 18 , the maximum detection distance of radar is $6 \mathrm{~km}$, missile has a maximum range of $3 \mathrm{~km}$, enemy UAV aerial index is 25 , the maximum detection distance of radar is $8 \mathrm{~km}$, Missile has a maximum range of $4 \mathrm{~km}$. Tactical maneuver to avoid the enemy's head sideways to make an attack, linear motion .

The simulation results were shown in Figure 5, 6 and Table 5.3, the method proposed in this paper was able to obtain a favorable position well, could make the maximum extent of destruction to enemy aircraft. 
Table 5. 3 Air Combat Situation Index and the Total Threat Index

\begin{tabular}{c|c|c|c|c|c|c|c|c}
\hline Enemy & \multicolumn{7}{|c|}{ EU } \\
\hline We & UAV1 & UAV2 & UAV3 & $P_{1}$ & UAV1 & UAV2 & UAV3 & $P_{2}$ \\
\hline$T_{a}$ & 0.342 & 0.436 & 0.425 & & 0.443 & 0.499 & 0.553 & \\
\hline$T_{r}$ & 2 & 3 & 2 & & 2 & 3 & 2 & \\
\hline$T_{v}$ & 1.200 & 1.000 & 0.800 & & 1.000 & 1.260 & 1.000 & \\
\hline$T_{S D}$ & 430.12 & 520.61 & 300.56 & & 413.68 & 553.62 & 315.25 & \\
\hline Thread & 171.82 & 192.62 & 163.52 & & 173.52 & 200.56 & 135.63 & \\
\hline$F$ & 200.45 & 200.33 & 200.78 & & 198.45 & 203.33 & 200.89 & \\
\hline$P$ & 0.425 & 0.460 & 0.299 & 0.382 & 0.526 & 0.562 & 0.512 & 0.579 \\
\hline
\end{tabular}

\section{Conclusion}

This paper studies three UAVs cooperative formation to attack an enemy aircraft placeholder technology, and takes the situation assessment and the threat model into consideration. At the same time, we establish the UAV kinematics equation and pseudo trace point trajectory. The simulation results show that three UAVs can trace the tracking pseudo point in real-time, operate threat assessment and destroy the enemy with the maximum extent of degree. It should be pointed out that, there is a bit shake from one site to the next site, so it needs to be improved. In addition, the effect, which is not considered in this paper of threat model and situation assessment to multi-UAV attacking occupying Technology in the influence of height. For example, "How to build a 3 dimensional environment with threat model and situation assessment" will be further researched and this will become a new topic for us to study.

\section{Acknowledgement}

Thank you for the personnel care for the guidance and enthusiasm about this paper ,that I learned a lot of from them, they give me a profound influence on my future research, in this pay tribute to them and sincere thanks . Finally, thanks to the members of the expert reviewers to guidance of my papers in their busy.

\section{References}

[1] Y. Tao, "Drone attacks on occupying automatic path generation study", China Aviation Institute of Computing Technology, (2009).

[2] K. D. Huang, D. Y. Zhou, C. H. Zhou and Yuji, "UCAV close combat strategies and methods placeholder", Northwestern Polytechnical University, vol. 8, no. 4, (2013), p. 35.

[3] P. H. Wang, C. S. Jiang, C. Yang, "Combat fighter fuzzy neural network footprint", Nan Jing University of Aeronautics and Astrona, vol. 8, no. 3, (2001), p. 83.

[4] Z. Q. Li and D. Y. Zhou, "For Target Variable Structure Guidance Law", Fire Control and Command Control, vol. 5, no. 5, (2009), p. 34.

[5] W. Q. Guo, "UAV formation controller design consistency theory", Harbin Institute of Technology, vol. 7, (2013).

[6] Y. P. Zhu, "Multiple UAVs coordinated attack Strategy", Nan Jing University of Aeronautics and Astrona, vol. 1, (2012).

[7] D. X. Wang, Z. Liu and J. C. Huang, "Air combat mission planning based on threat assessment", Fire Control and Command Control, vol. 12, no. 36, (2007).

[8] S. L. Zhou, C. Zhou and W. G. Zhang, "UAV flight formation based predictive control method", Naval Aeronautical Engineering Institute, vol. 26, no. 6, (2011),pp. 601-605.

[9] Y. F. Dong, J. L. Feng and H. X. Zhang, "Tactical Decision Methods for Multi- aircraft Air Combat Simulation collaboration”, Journal of System Simulation, vol. 14, no. 16, (2002), pp. 723-724.

[10] F. Austin'Giro Carbone, “Automated Maneuvering Decisions for air-to-air Combat”, (1987). 
[11] R. Zhou, W. M. Wu and G. W. Luo, "Autonomous decentralized multi- UAV cooperative control”, Journal of Aeronautics, vol. 29, (2008), pp. 26-32.

[12] R. Wise, "UAV Control and Guidance for Autonomous Cooperative Tracking of a Moving Target", University of Washington.

\section{Authors}

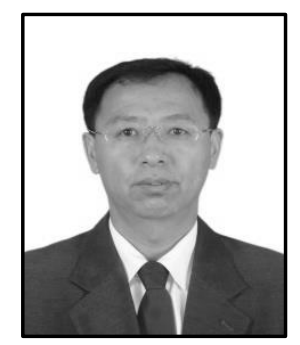

Yibo Li. Born in 1963, he received master degree from Nanjing University of Aeronautics and Astronautics in 1988 and doctor degree from Northeastern University in 2003. He became a teacher in Shenyang Aerospace University since 1988. His main research interests are image processing, pattern recognition, flight control, etc.

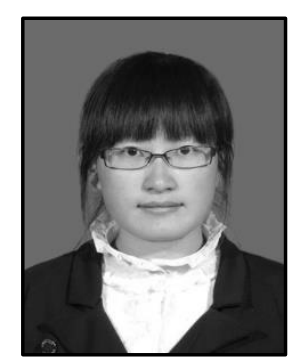

Xiaonan Guo. Born in 1989, he received bachelor degree from Liaoning Shenyang Aerospace University in 2008. His main research interests are air combat and autonomous control.

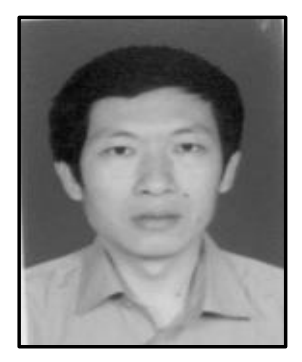

Wei Chen. Born in 1964, he received master degree from Northwestern Polytechnical University in 1988. He became a chief designer in AVIC Chengdu aircraft industrial (group) CO,LTD since 1988. His main research interests are aircraft control, navigation.

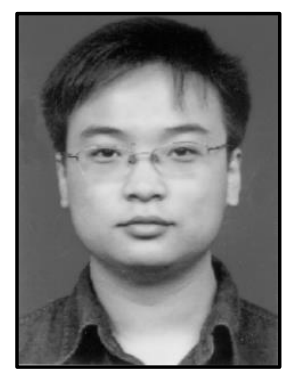

Yi Wang. Born in 1980, he received master degree from Beijing University of Aeronautics and Astronautics in2012. He became a technical center director designer in AVIC Chengdu aircraft industrial (group) CO,LTD since 2003. His main research interests are UAV flight control and management, With flying UAV formation control, Self-control,etc. 
International Journal of Control and Automation

Vol. 8, No. 1 (2015) 